Proc. Indian Acad. Sci. (Earth Planet. Sci.), Vol. 104, No. 2, June 1995, pp. 257-271.

(C) Printed in India.

\title{
Estimation of surface temperature from MONTBLEX data
}

\author{
K NARAHARI RAO \\ Centre for Atmospheric Sciences, Indian Institute of Science, Bangalore 560012, India
}

\begin{abstract}
It is observed that the daily mean temperature of the soil is linear with depth and the variation of the temperature is sinusoidal with a period of a day. Based on these observations the one-dimensional heat conduction equation for the soil can be solved which gives the amplitude and phase variation of the temperature wave with depth. Given the temperature data at three levels below the surface, the amplitude and phase variation and hence the surface temperature variation over the day are estimated. The daily mean temperature of the surface is estimated from linear extrapolation of the daily means at the three levels below the surface. Estimated values of soil thermal diffusivity show a subtantial change after sudden and heavy rains.
\end{abstract}

Keywords. Soil and surface temperature; diurnal variation; phase and amplitude of temperature waves; thermal diffusivity.

\section{Introduction}

Because of the importance of the surface layer in determining the eddy fluxes of mass, momentum and energy, the Monsoon Trough Boundary Layer Experiment (MONTBLEX) conducted during the monsoon of 1990 gave particular importance to the surface layer measurements. The locations chosen for such observations lie along the trough, and are:

- IIT Kharagpur (KGP),

- Banaras Hindu University at Varanasi (BHU),

- IIT Delhi (DEL), and

- CAZRI at Jodhpur (JDP).

$30 \mathrm{~m}$ masts were erected at each site with the required instrumentation at six levels $(1,2$, $4,8,15$ and $30 \mathrm{~m}$ ) above ground level to measure vertical and horizontal velocities, wind direction, temperature and humidity (Rudra Kumar et al 1991).

The soil temperature was measured at three levels $(0.1,0.2$ and $0.3 \mathrm{~m})$ below the ground using resistance thermometers of 12.5 microns diameter platinum wire encapsulated in ceramic. These were sampled at $1 \mathrm{~Hz}$, averaged over one minute or three minutes and stored on audio cassette tape through a Campbell data logger. For a proper analysis of these data, it is important to have precise information about surface parameters such as roughness length, temperature, moisture etc. To estimate the surface temperature, which is the purpose of the present analysis, we use 30 minute averages of soil temperature obtained from the logged data. 


\section{Equations for the surface layer}

The wind and temperature profiles in the surface layer are governed by the following equations:

$$
\begin{aligned}
u(z) & =\left(u_{*} / \kappa\right)\left(\ln \left(z / z_{0}\right)-\psi_{M}(z)\right), \\
\theta(z)-\theta_{0} & =\left(0 \cdot 74 \theta_{*} / \kappa\right)\left(\ln \left(z / z_{0}\right)-\psi_{H}(z)\right),
\end{aligned}
$$

where $u(z)=$ mean wind speed at height $z, \mathrm{~m} / \mathrm{s}$

$$
\begin{array}{ll}
u_{*} & =\text { characteristic (friction) velocity, } \mathrm{m} / \mathrm{s} \\
z & =\text { vertical height, metres } \\
z_{0} & =\text { roughness length, metres } \\
\kappa & =\text { von Karman constant }=0.41 \\
\theta(z) & =\text { temperature at height } z, \text { degrees } \\
\theta_{*} & =\text { characteristic temperature, degrees } \\
\theta_{0} & =\text { surface temperature, degrees } \\
\psi_{M}, \psi_{H} & =\text { correction factors for } u \text { and } \theta \text { in the log law. }
\end{array}
$$

The surface temperature, which is not an easy quantity to measure, was not directly measured during MONTBLEX. However, since the soil temperatures have been recorded at three levels below the ground, $\theta_{0}$ can in principle be inferred from this data.

\section{Evaluation of surface temperature}

We first assume that temperature is governed by the one-dimensional heat conduction equation in the soil,

where

$$
\partial \theta / \partial t=D \partial^{2} \theta / \partial z^{2}
$$

$$
\begin{aligned}
& D=K / \rho C, \text { thermal diffusivity of the soil, } \mathrm{m}^{2} / \mathrm{s} \\
& \rho=\text { density of soil, } \mathrm{kg} / \mathrm{m}^{3} \\
& C=\text { specific heat of soil, cal kg-1 } \operatorname{deg} C^{-1} \\
& K=\text { conductivity of the soil, cal m-1 } \mathrm{s}^{-1} \operatorname{deg~} C^{-1}
\end{aligned}
$$

We can in principle obtain the thermal diffusivity using equation (3). One approach is the following: The time derivative of temperature at the middle level can be obtained from

$$
\partial \theta / \partial t=(\theta(t+1)-\theta(t-1)) / 2 \Delta t
$$

The second derivative at the middle level is obtained from the three temperature measurements at $z_{1}, z_{2}$ and $z_{3}$ as:

$$
\partial^{2} \theta / \partial z^{2}=\left(\theta\left(z_{3}\right)-2 \theta\left(z_{2}\right)+\theta\left(z_{1}\right)\right) /(\Delta z)^{2},
$$



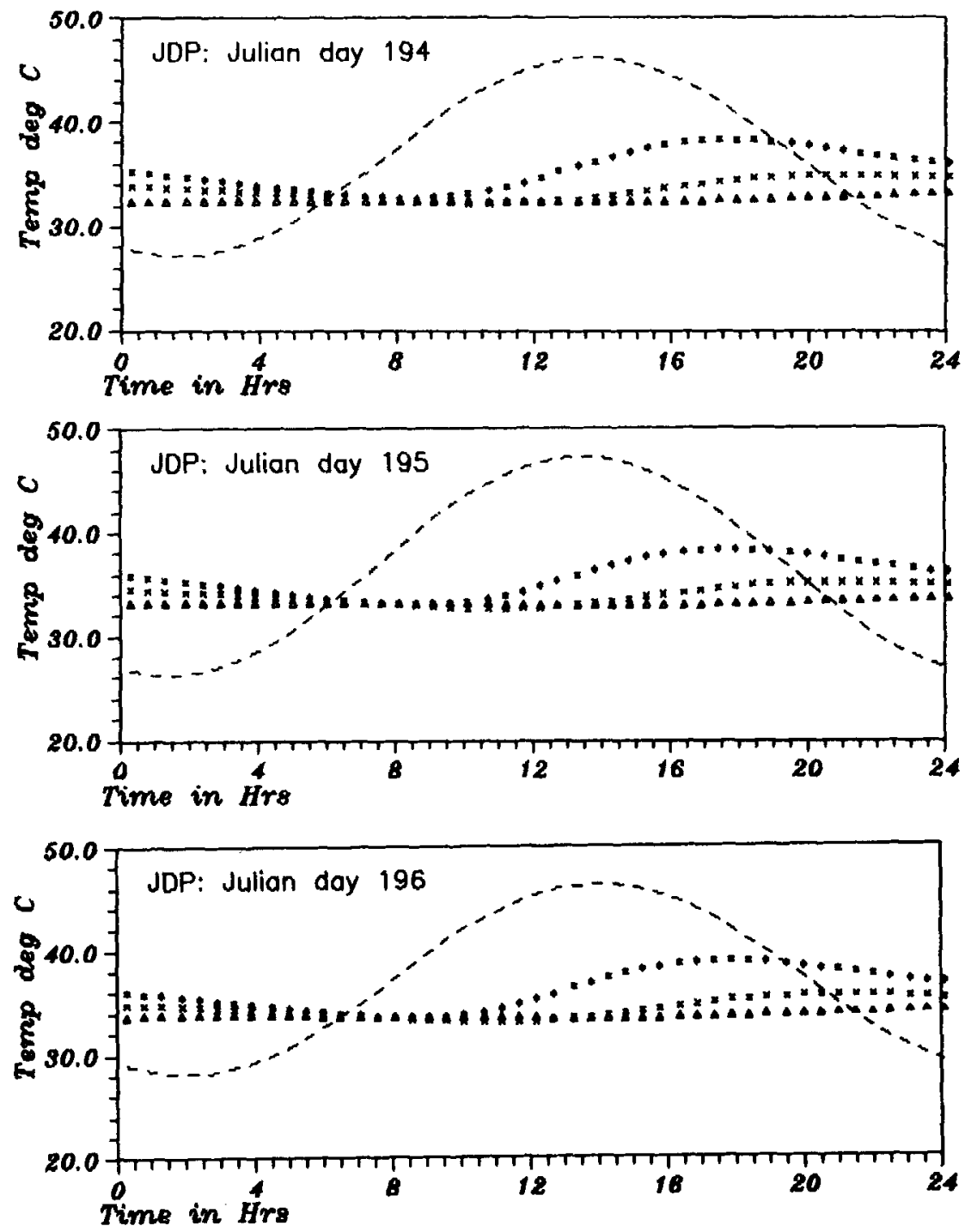

Figure 1. Variation of temperature over the day $\left(^{*}-0.1 \mathrm{~m}, \mathrm{x}-0.2 \mathrm{~m}, \Delta-0.3 \mathrm{~m}\right.$ and $-\ldots$ surface).

and substituting from equations (4) and (5), $D$ can be evaluated from equation (3). Once $D$ is known, applying the equations to the first level it is possible to evaluate the surface temperature $\theta_{0}$.

Computations however show that, many times during the day, the sign of $\partial \theta / \partial t$ evaluated as above does not agree with that of $\partial^{2} \theta / \partial z^{2}$, implying a negative coefficient of diffusion! The derivatives depend heavily on the accuracy of the temperatures measured, which in turn depends on the calibration constants and drifts. The difficulties of obtaining derivatives numerically are well known, and it was felt that this method is not the appropriate one to use here in the present situation.

Measurements show that the temperature variation of the soil is approximately a sine wave with a period of one day at all three levels below the surface, with a phase 


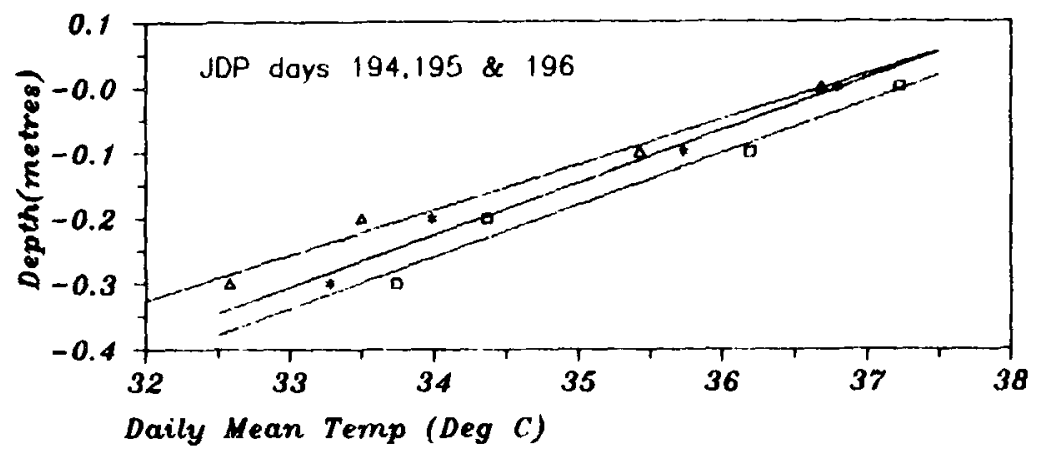

Figure 2. Variation of daily average temperature with depth at JDP. ( $\Delta$ - day $194 ;^{*}$ - day 195 and $\square$ - day 196).
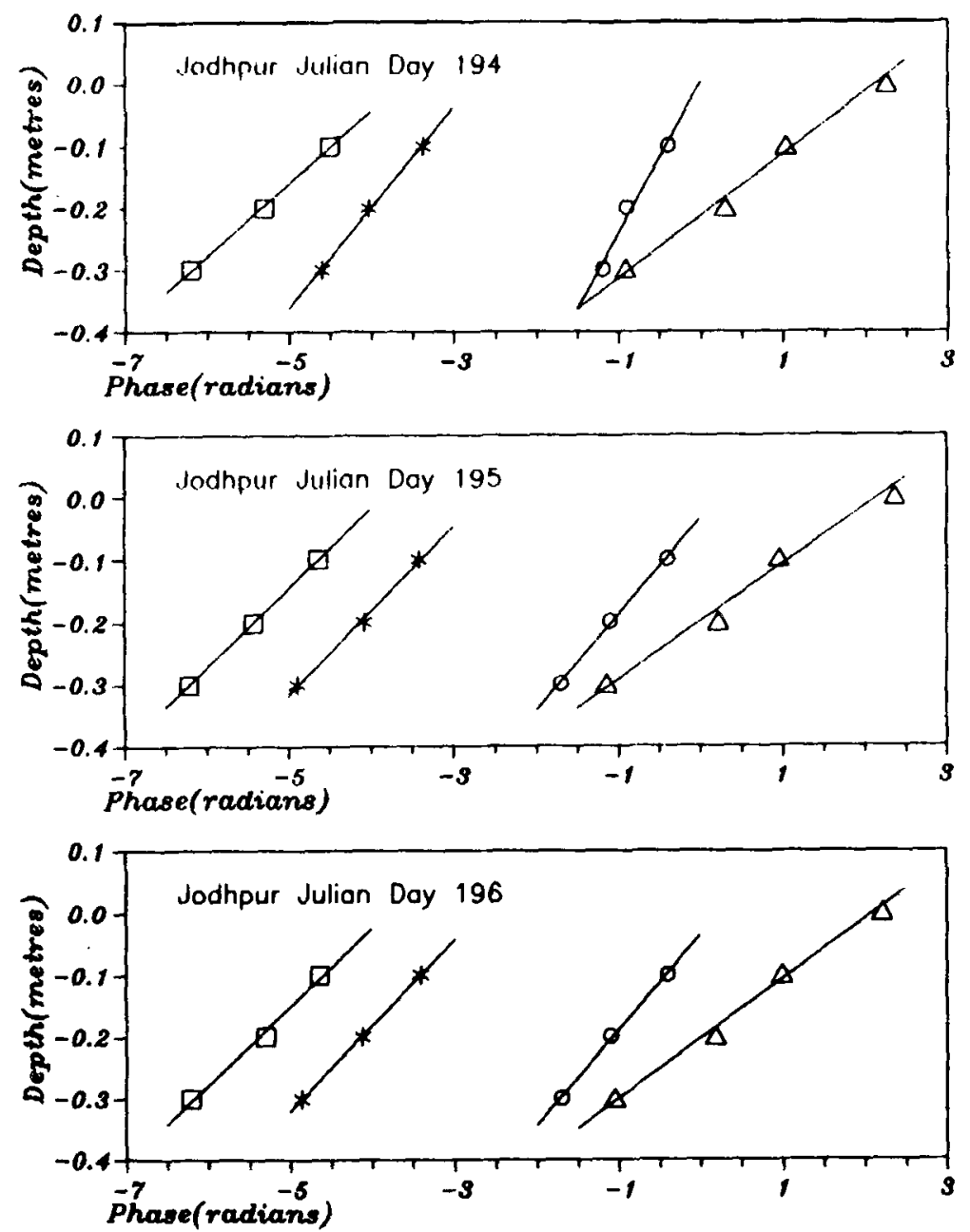

Figure 3. Variation of the phase with depth $\left(\square\right.$-beginning, ${ }^{*}$-mid, o-maximum and $\Delta$ - logarithm of the amplitude). 


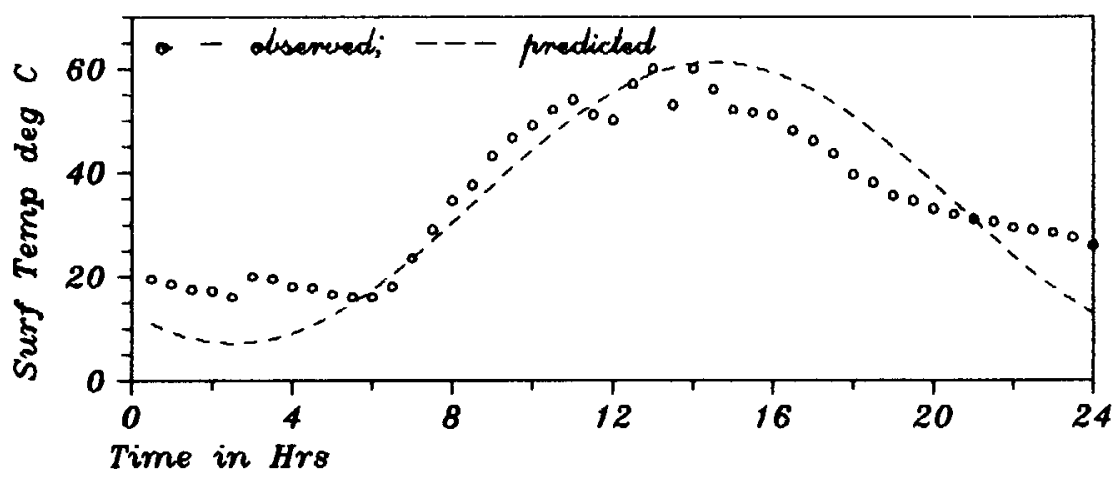

Figure 4. Comparison of computed and measured surface temperatures-data from Pune, 4th-6th May 1992.

Table 1. Variation of alpha.

\begin{tabular}{lrrrr}
\hline & BHU & DEL & JDP & KGP \\
\hline No of pts: & 60 & 43 & 64 & 54 \\
Mean alpha: & 3.54 & 6.82 & 6.63 & 4.98 \\
Std. Dev.: & 1.31 & 1.90 & 1.77 & 1.68 \\
Limit for 95\% confidence: & 0.34 & 0.59 & 0.44 & 0.46 \\
\hline
\end{tabular}

difference between different levels (figure 1). With this condition, the solution of equation (3) can be obtained as (Kirkham and Powers (1972)):

$$
\theta(z, t)=\bar{\theta}(z)+\tilde{\theta} e^{-\alpha z} \sin (\omega t-\alpha z),
$$

where $\bar{\theta}$ is the mean temperature at depth $z$ over the day, $\tilde{\theta}$ is the amplitude of the sine wave at the surface,

$$
\begin{aligned}
& \alpha=\sqrt{(\omega / 2 D)} \text { and has the units metre }{ }^{-1}, \\
& \omega=2 \pi / P \text { in radians } / \mathrm{hr}, \text { and } \\
& P=24 \mathrm{hrs}, \text { the period of the sine wave. }
\end{aligned}
$$

It is also observed that the mean temperature over the day varies linearly with depth (figure 2). Hence, a value of $\bar{\theta}$ at $z=0$ can be obtained from extrapolation, and is shown there in figure 2 .

In equation (6), one finds that the amplitude decreases like $e^{-x z}$ and the phase of the sine wave shifts by $\alpha z$ with depth $z$. Therefore, from the measurements, $\alpha$ can be determined in two ways - one from the amplitude and the other from the phase.

In the first method the maximum and minimum of the temperature wave, the difference between which gives twice the amplitude, is determined. With temperature measurements at three levels below the ground level, knowing that the amplitude varies like $e^{-\alpha z}$, we can evaluate $\alpha$. Figure 3 shows the variation of the logarithm of the amplitude with depth; the slope of the straight line in these plots gives $\alpha$. The value shown at $z=0$ is from extrapolation. It may be noted that the amplitude at the lowest 


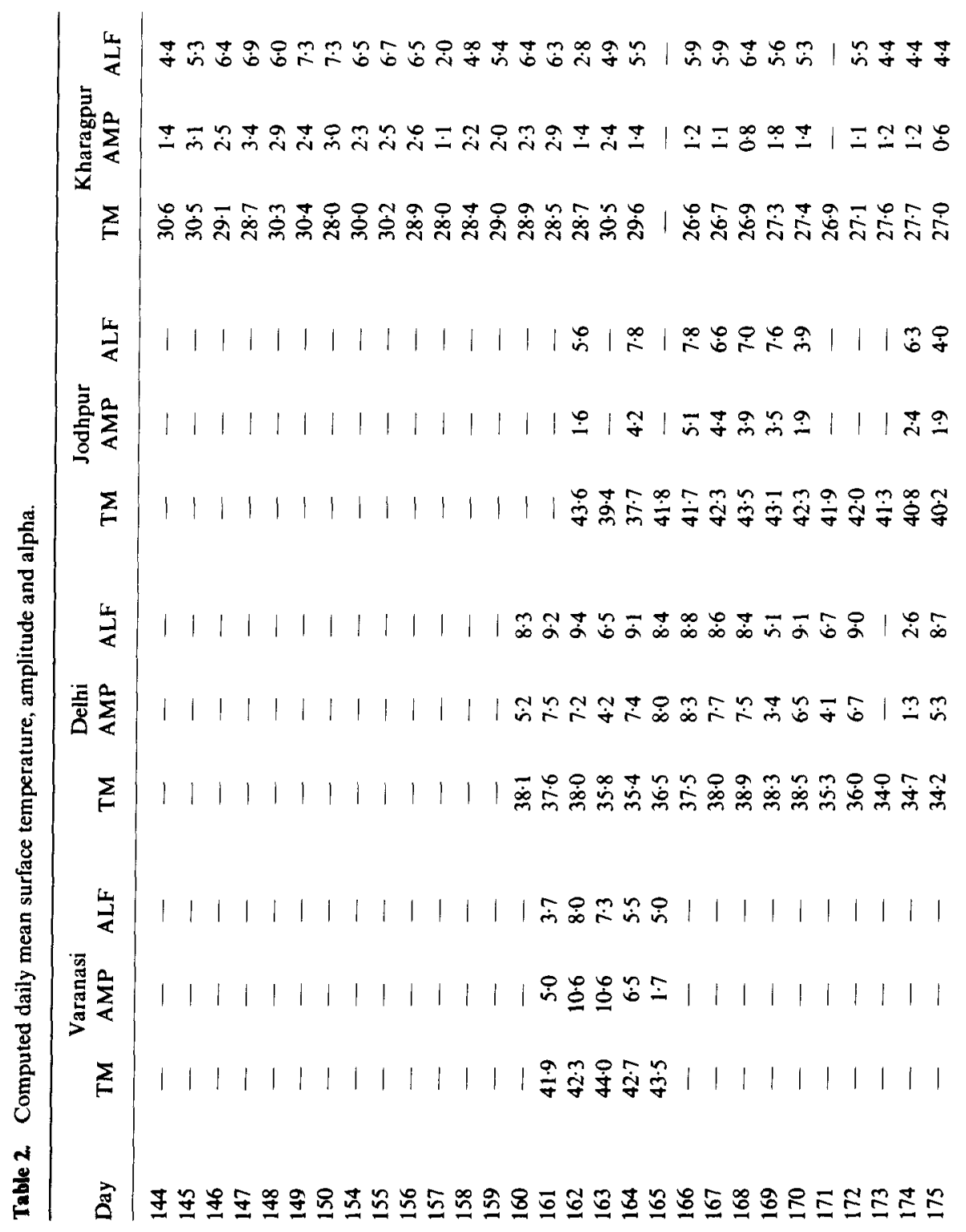


| 1 |

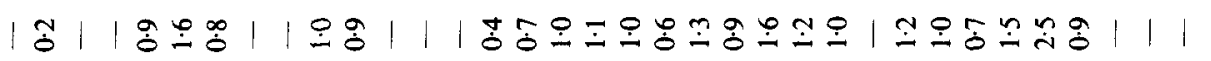

| 完 $\mid$ |

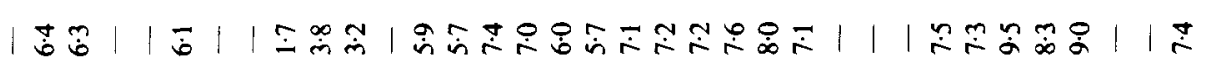

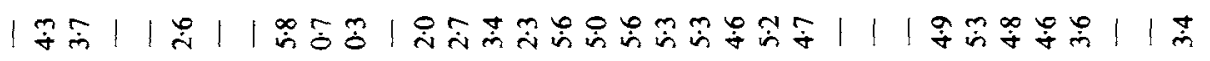

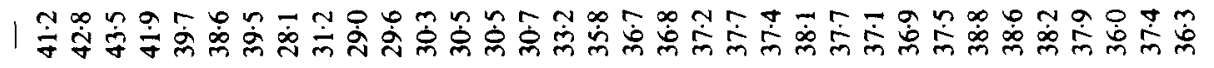

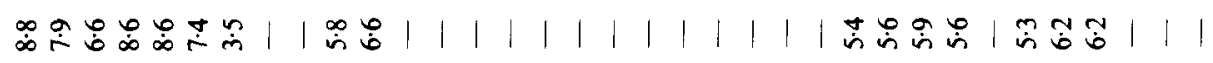

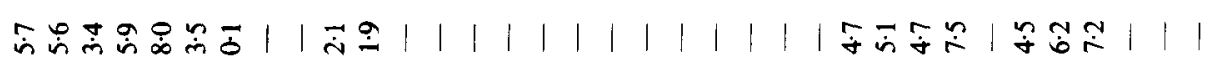

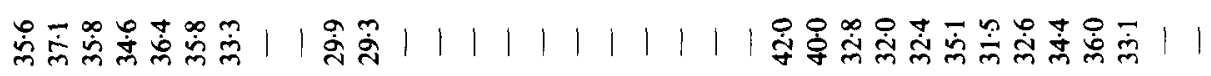
| $|1|$ | | | | | | | |

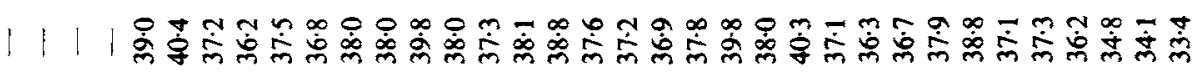

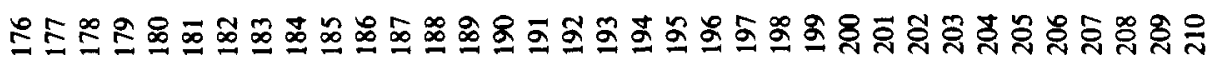




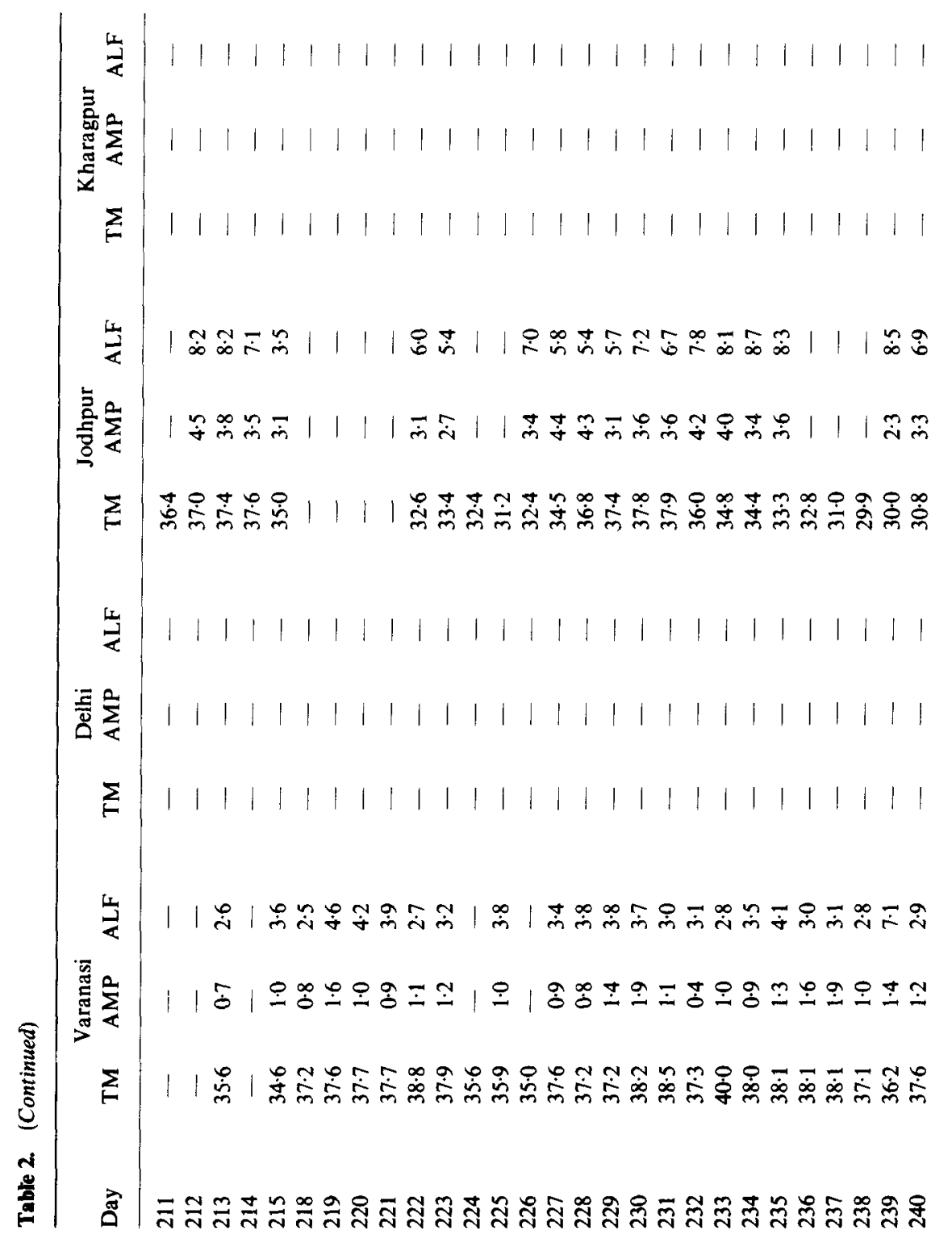




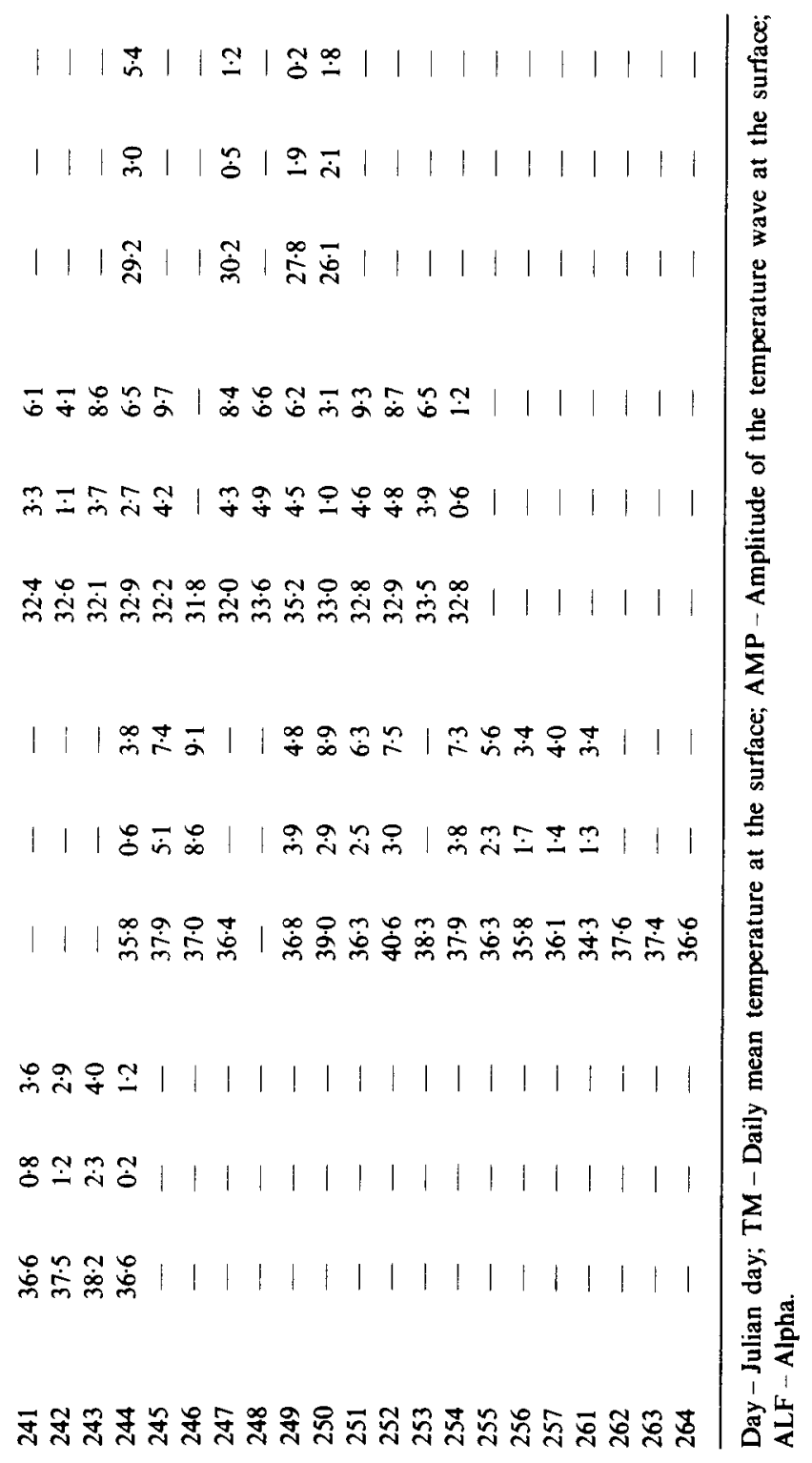


level does not fit into the trend. This may be due to difficulty in accurate evaluation of a small amplitude or in the calibration constant for that sensor.

The phase differences between the three waves below the ground can be found in four different ways. Since the sine wave has a period of a day, we can determine the phase from the point where the wave crosses the mean. This happens at two points during every cycle. However, it is found from experience that the crossings around midnight are more difficult to determine than the middle one, which is simply due to the fact that the wave crosses the mean with a distinct positive slope around midday. Figure 3 shows the variation of phase determined from both the midnight and midday crossings with depth. The fourth method is to determine the time when during the day the temperature

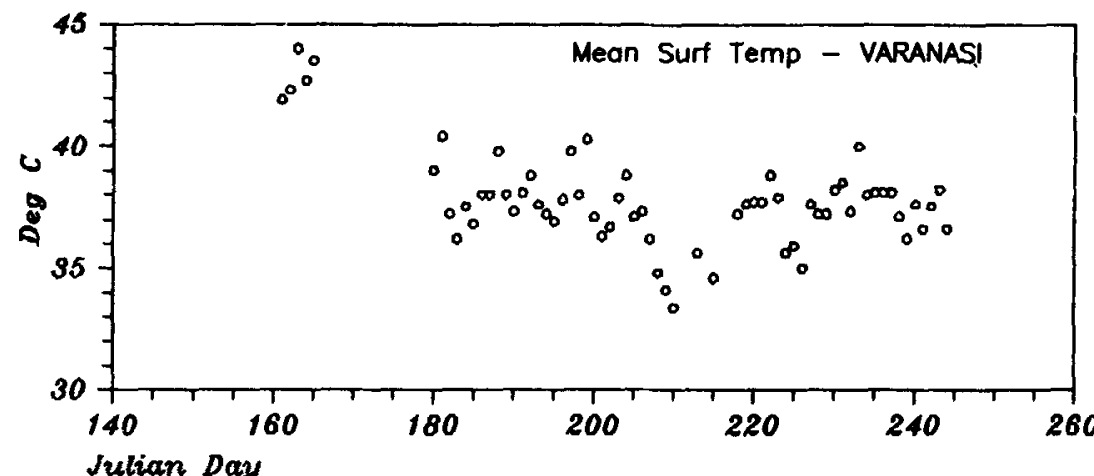

(a)

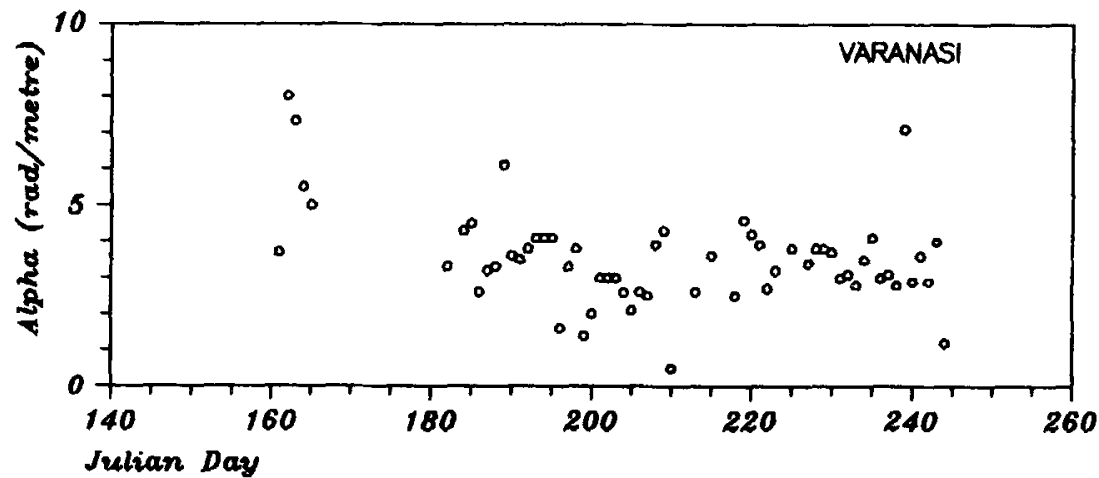

(b)

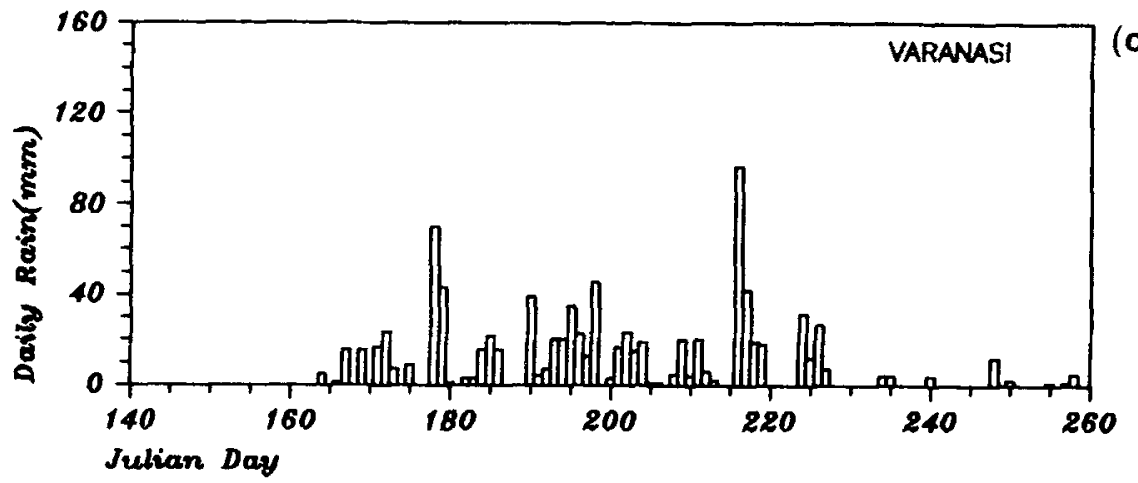

Figure 5. (a) Surface mean temperature; (b) $\alpha$; and (c) rain at BHU. 


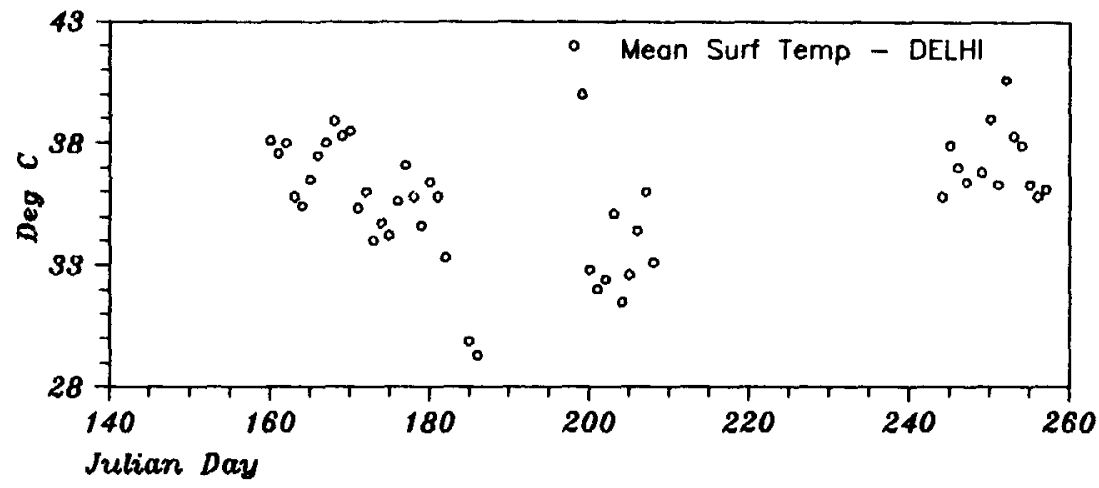

(a)

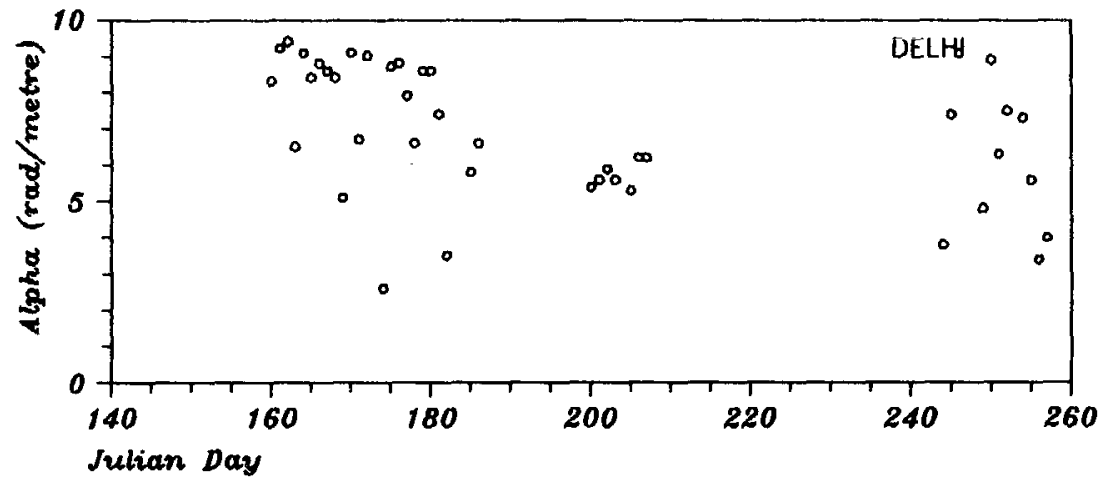

(b)

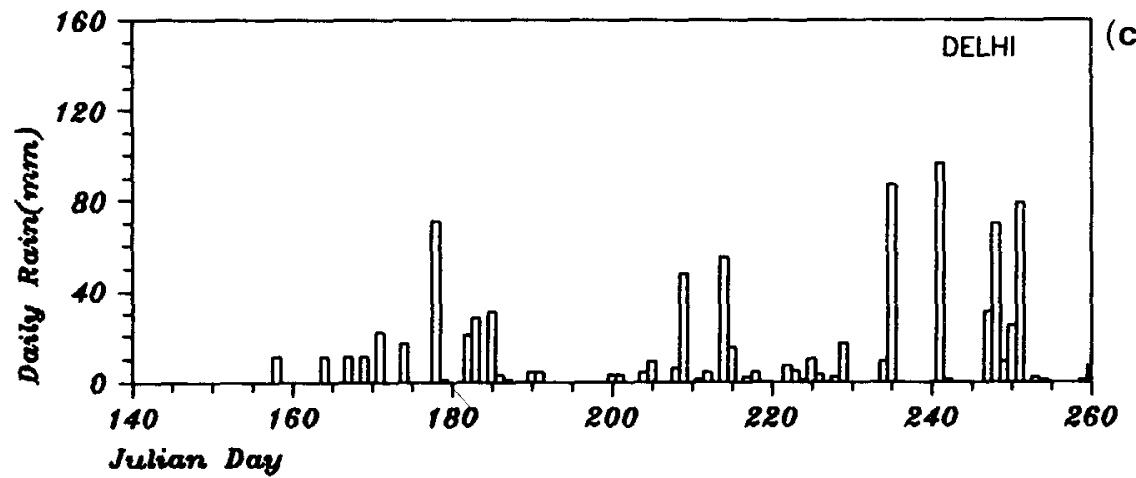

(c)

Figure 6. (a) Surface mean temperature; (b) $\alpha$; and (c) rain at DEL.

waves have a maximum, which is also a measure of the phase of the wave. In principle, this is more difficult as the time at which the maximum occurs is not sharply defined, since a sine wave has zero slope at this point. However, it can be seen from figure 3 that this method also gives a reasonable estimate of $\alpha$.

It may be noted here that the value of the temperature measured within the soil depends on the calibration constant and drift (if any) of the instrument; so does the amplitude. On the other hand, the phase does not depend on either of the above, and hence should actually give the most reliable information about $\alpha$.

It is possible that $\alpha$ cannot be estimated by all the four methods mentioned above, mainly because of the discontinuity in data at some stretches during the day. $\alpha$ obtained 
from different methods is averaged for further analysis. If $\alpha$ cannot be estimated by two methods at least, such data has been discarded.

Knowing the mean temperature at three levels below the ground a straight line can be fitted through those three points and extrapolated to get the mean temperature at the surface (figure 2). The phase difference and the amplitude of the surface temperature can be computed from $\alpha$ and the temperature data at the first level below the surface. The temperature of the surface for the whole day can be computed. The dotted line in figure 1 shows the result. Taking into account the longer day at Jodhpur during that time, this temperature wave crosses the mean around the same time delay after sunrise and sunset.

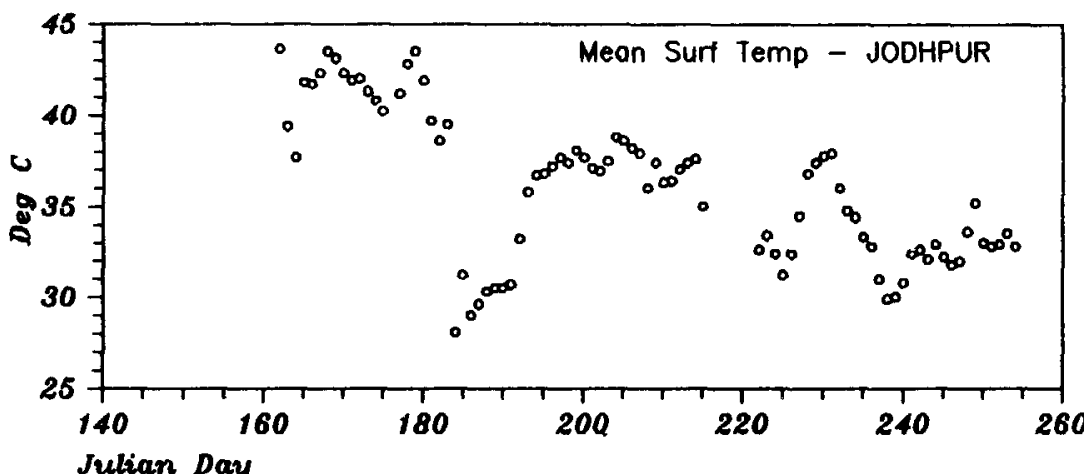

(a)

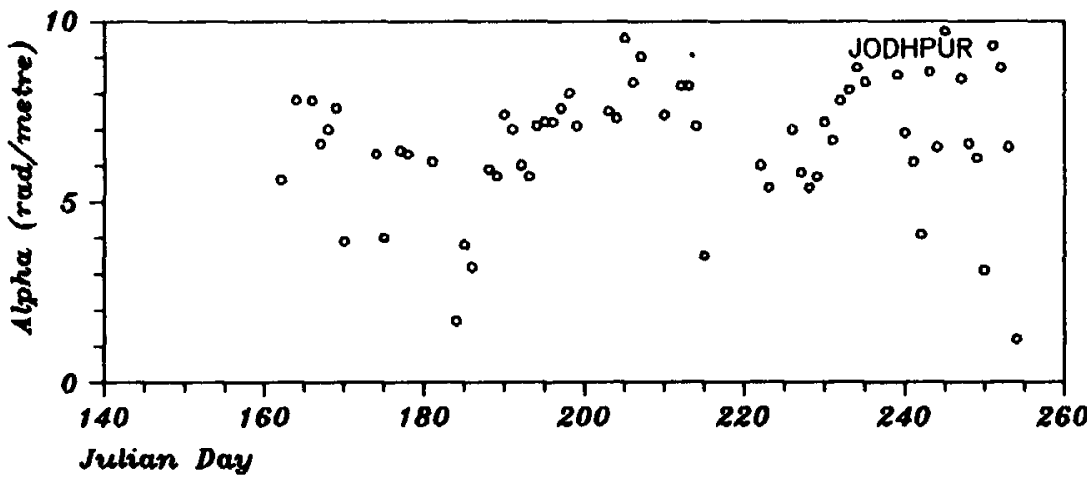

(b)

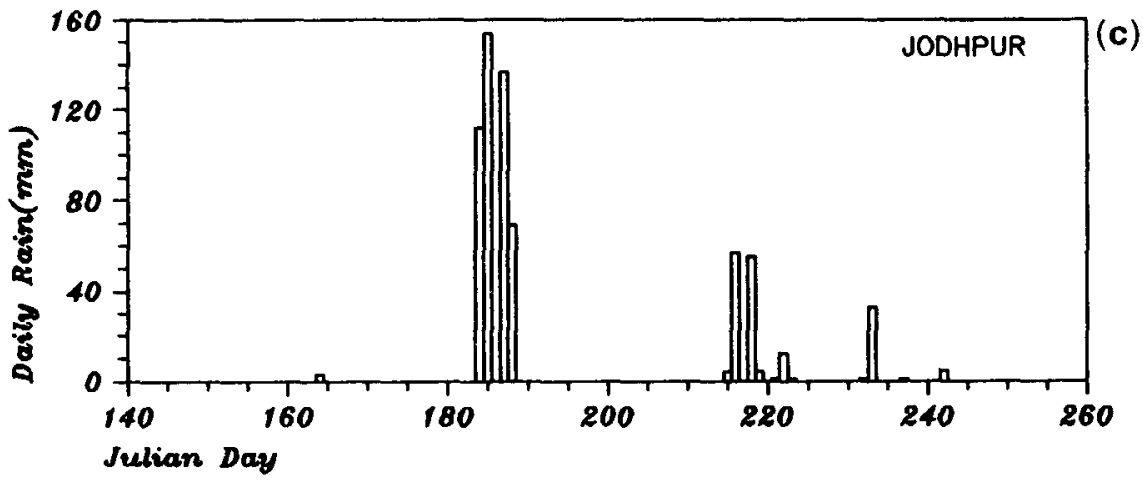

Figure 7. (a) Surface mean temperature; (b) $\alpha$; and (c) rain at JDP. 


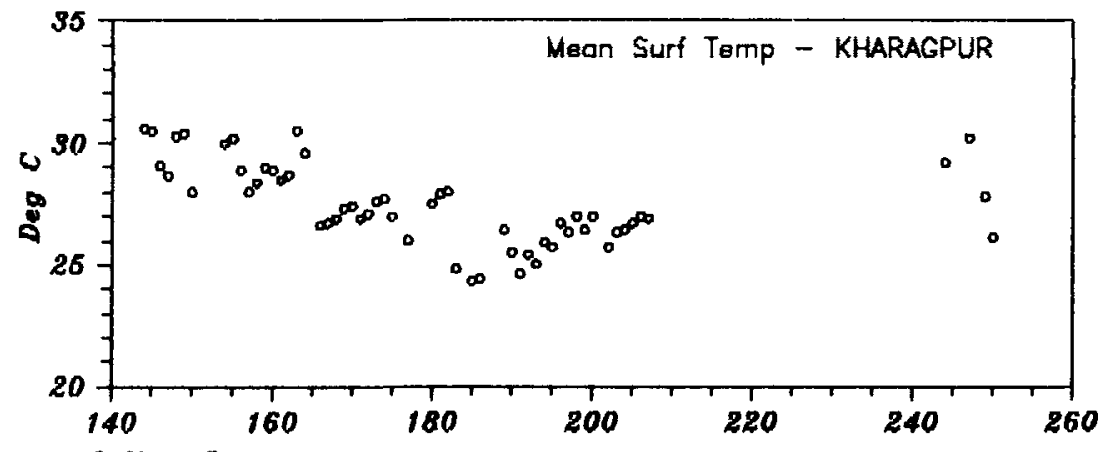

Juliar Day

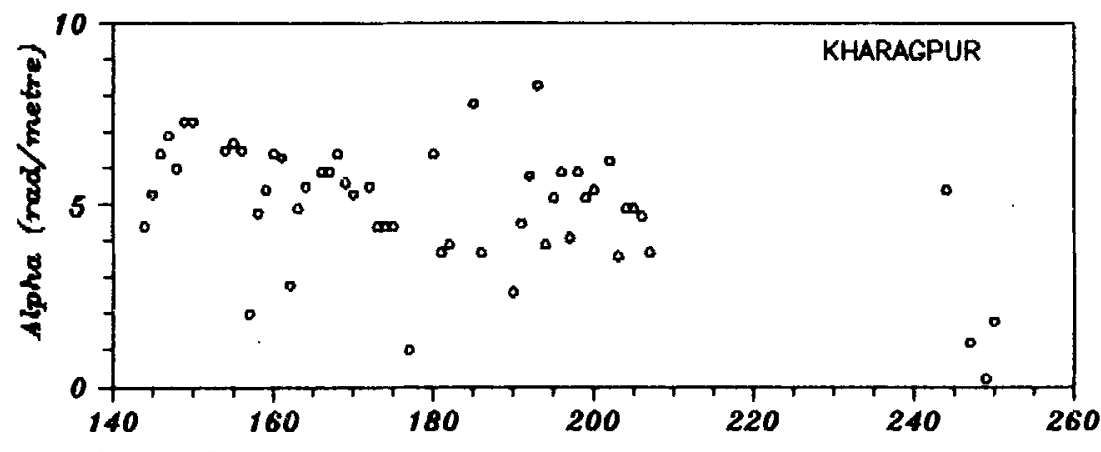

(b)

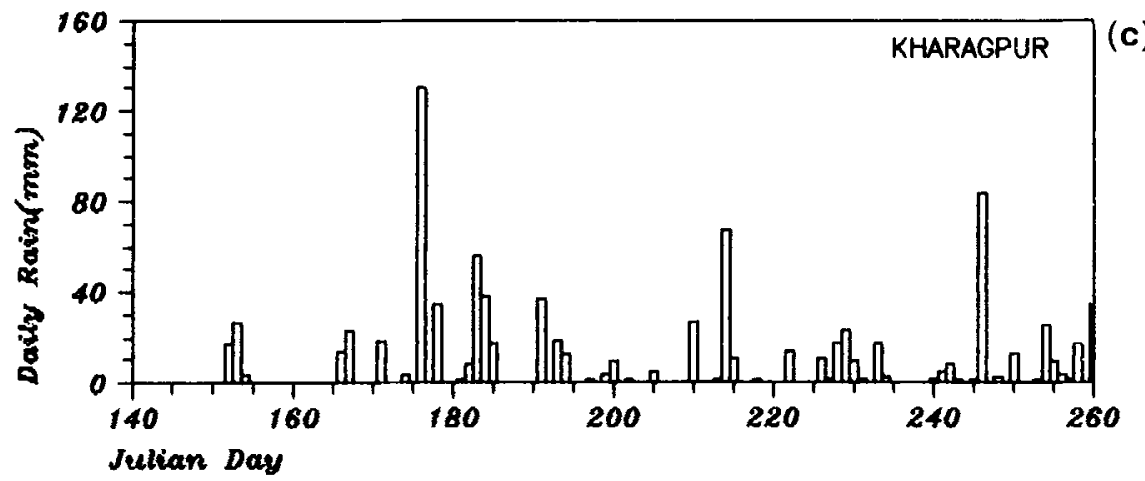

Figure 8. (a) Surface mean temperature; (b) $\alpha$; and (c) rain at KGP.

Figure 4 gives the observed surface temperature along with the surface temperature computed as explained above from the data at three levels below the ground during 4th to 6th May 1992 at Pune (kindly supplied by Dr. Verneker, IITM). The agreement is very good, giving us confidence in the present procedures for obtaining the surface temperature.

\section{Conclusions and recommendations}

Table 1 gives the mean, standard deviation and $95 \%$ confidence levels for the mean of $\alpha$ for all the four stations. The values are seen to be different for different stations. The spread seems to be large (about 25 to $30 \%$ of the mean). The values of the thermal 


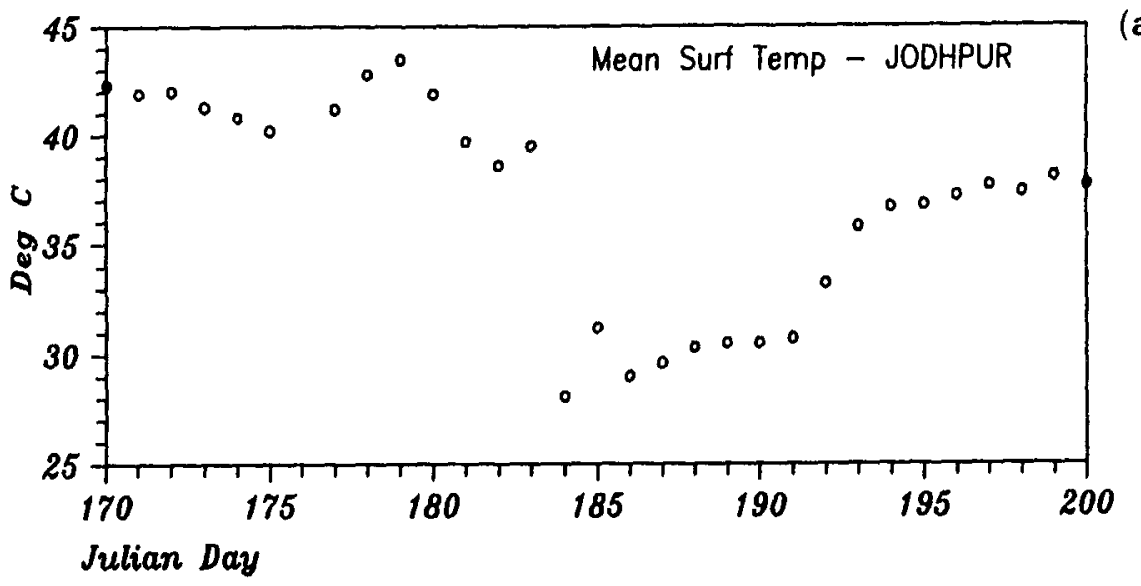

(a)

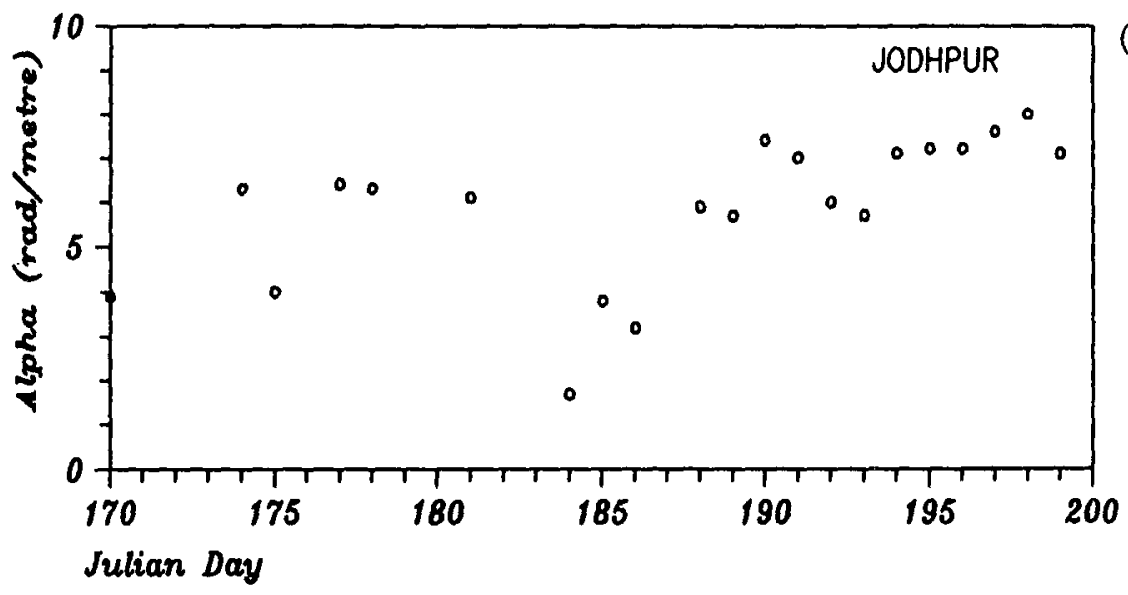

(b)

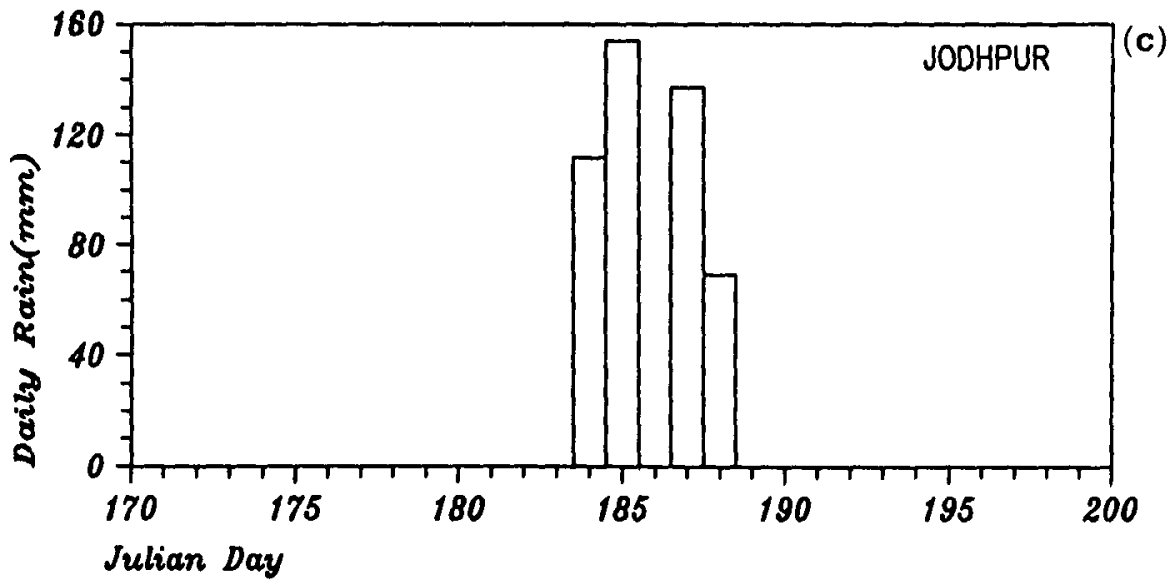

Figure 9. (a) Surface mean temperature; (b) $\alpha$; and (c) rain at JDP around a heavy rainfall. 
diffusivity calculated from $\alpha$ are in the range (about $0.1 \times 10^{-6}$ to $1.3 \times 10^{-6} \mathrm{~m}^{2} / \mathrm{s}$ ) reported by Kirkham and Powers (1972).

The mean surface temperature over the day, the amplitude of the temperature wave at the surface and $\alpha$ are computed over the period of the experiment and are given in table 2 which can be used for getting the surface temperature at any time of the day. The daily mean surface temperature, $\alpha$ and the rainfall at all the four stations are presented in figures 5 to 8 .

It can be seen from the Jodhpur data (figure 7) that the $\alpha$ and the mean surface temperature change appreciably following heavy rains. To illustrate this more clearly, figure 9 shows the period of such an event at Jodhpur. At this station, the mean $\alpha$ over the full duration of the experiment is $6.63 \mathrm{rad} / \mathrm{m}$ with a standard deviation of 1.77 $\mathrm{rad} / \mathrm{m}$. Following rain on day 183 , the value of $\alpha$ drops to about $1.7 \mathrm{rad} / \mathrm{m}$ on day 184 and the mean surface temperature over the day falls drastically from $39.5^{\circ} \mathrm{C}$ on day 183 to $28.1^{\circ} \mathrm{C}$ on day 184 . It is observed during the experiment that the air temperature during the early hours of day 184 was around $25^{\circ} \mathrm{C}$. From the data, the estimated probability of $\alpha$ attaining this low value is less than $1 \%$. A similar trend was observed around day 220. It is therefore clear that the thermal diffusivity and the surface temperature of the soil must have changed substantially after sudden, very heavy rains.

At other sites, the rainfall was almost uniformly spread over the period of experiment; so no sudden changes can be observed. However, as long as the temperature waves are sinusoidal and the mean temperatures are linear with depth, the present analysis should still hold good.

\section{References}

Kirkham D and Powers W L 1972 Advanced Soil Physics (Wiley Interscience)

Rudra Kumar S, Srinivasan H P, Srikrishna R, Ameenulla S, Prabhu A (1991) Available tower data from MONTBLEX 1990, Rẹp 91 M D 1 CAS, IISc, Bangalore 12. 